\title{
Gravity-Independent and Gravity-Related Inertia Fields
}

\author{
Robert L. Shuler \\ NASA Johnson Space Center, Houston, Texas, United States \\ Email: robert.1.shuler@nasa.jsc or robert@mc1soft.com
}

\begin{abstract}
This paper first assesses under what conditions the Higgs field has "no deep connection" to gravity, i.e. it is gravity-independent, and also whether it has a connection with or conflicts with other proposed inertia-causing fields such as the vacuum-reaction force. Then it develops a classical consistent field strength (CFS) framework to support analysis of inertia fields that are gravity-related, which seems likely in the case of vacuum-reaction inertia. The framework can produce important exact solutions to Einstein's field equation. When used with alternative formulations it guarantees equivalence and conservative fields over a wide range of field choices from traditional metric spacetime to background-embedded to emergent space-time. A short worked-out gravity-related inertia field example is given. New perspectives on spin and self-gravitation issues are discussed.
\end{abstract}

Keywords: Inertia, gravity, space-time, general relativity, Higgs field, vacuum energy

\section{Introduction}

Since the 1960s physicists have been engaged in developing theories of rest mass, or inertia, at the quantum level. The most widely followed such theory is the Higgs mechanism [1], boosted by the recent discovery of its boson [2]. By interactions with the non-zero Higgs field, particles which would otherwise be traveling at the speed of light appear to manifest some of their energy as rest mass. There are at least superficially differing perspectives on the relation - or not - between particle physics and gravity. For example, the chief U.S. theoretician for the European Organization for Nuclear Research (CERN) says there is "no deep connection" between Higgs and gravity. ${ }^{1}$ By contrast Weinberg, Nobel laureate particle physicist, expressed frustration in the preface to his textbook on gravity that the "deep connection" between gravitational and particle physics was obscured, and voiced that inertia and equivalence are a legitimate starting point for the discussion of gravity [3]:

[In] textbooks geometric ideas were given a starring role, so that a student who asked why the gravitational field is represented by a metric tensor [or] freely falling particles move on geodesics [or] the field equations are generally covariant would come away with an impression this [was because] space-time is a Riemannian manifold ... This was Einstein's point of view ... [but] the geometric approach has driven a wedge between general relativity and the theory of elementary particles. ... [We no longer] expect that the strong, weak and electromagnetic interactions can be understood in geometrical terms, and too great an emphasis on geometry can only obscure the deep connections between gravitation and the rest of physics. ... In place of Riemannian geometry, I have based the discussion of general relativity on a principle derived from experiment: the Principle of the Equivalence of Gravitation and Inertia.

A second quantum theory of inertia was developed first in the Soviet Union in 1968 then from 1993 in the U.S. with a reaction force in the polarized vacuum [4]. This one has a smaller number of backers, but they do relate it to gravity, at least as far as time dilation, from which only weak field agreement has been be obtained [5-6]. Meanwhile notions of the scope of the Higgs theory were gradually broadening beyond just the $\mathrm{W}$ and $\mathrm{Z}$ bosons. With data coming in, the Higgs field is confirmed and even if its properties are not fully known they are the subject of one of the best organized and well financed experimental programs in history. So, we expect further data.

1 M. Strassler, "Why the Higgs and Gravity are Unrelated," https://profmattstrassler.com/2012/10/15/why-thehiggs-and-gravity-are-unrelated/ , posted Oct. 15, 2012, retrieved Oct. 26, 2016. 
The first objective of this paper is to briefly clarify the conditions under which there is indeed no deep connection between Higgs and gravity, which are quite restrictive, and though they were perhaps not satisfied in the original concept, they are consistent with the developing expanded notion of the Higgs, which has the theoretical flexibility to accommodate the conditions.

The second and larger objective of this paper is to address the more difficult problem of the polarized vacuum, and to ask if there is room for two fields or must we decide. For example time dilation or the speed of light that cannot have causes or regulating mechanisms that are independent. A two-factor classical framework is developed which accommodates a gravity-related inertia field along with the Higgs field in non-conflicting roles, and identifies but does not provide needed mechanisms.

\subsection{Gravity-Independent Inertia Field Restrictions}

In general relativity (GR) the "field" is space-time, and use of some other intermediate field is not desirable. Matter couples only to the metric, though other fields may influence the metric. Space-time determines the speed of light both locally and in a coordinate velocity sense. GR is so well backed by empirical data there is no need to change its views.

To have "no deep connection" to gravity an inertia-causing field must, oddly enough, not couple directly to matter. How can it avoid that if it "causes" rest mass? It must do so by changing one thing into another without affecting the way either of them couple to the space-time metric. In the case of the Higgs field, the trajectories of light speed particles are interrupted by interactions which change spin and direction, analogous to confining light. Due to their momentum and energy, photons have inertia and are "heavy" if so confined [7-8]. The author wishes to comment regarding popular "metaphors" that in his personal view the Miller cocktail party metaphor [9] tends to evoke mass-lending, and prefers the less popular Penrose zig-zag metaphor [10] which does not, though of course both are particle oriented and fail to capture the nuance of wave excitations.

The second requirement is that a gravity-independent inertia field should not regulate the speed of light. It is perhaps less obvious why an inertia-causing field might regulate the speed of light. This will become evident later in the second part of our development, so for now we just state the condition.

\section{$1.2 \quad$ Gravity-Related Inertia Field General Characteristics}

In the second part, we take the opposite position, that an inertia field regulates not just the apportionment between rest mass and light speed momentum, but that it regulates inertia itself, the resistance to change in state of motion that is responsible for momentum. In that case we will argue below that it must also regulate light speed, which is a function of gravity. Therefore either gravity must be the regulator of momentum-mass, or the inertia field must be responsible for gravitational effects. There are difficulties with either side of the proposition in traditional formulations, which have contributed to the lack of an inertia-gravity framework to date.

From the GR perspective, rest mass is invariant. Einstein said in a 1912 paper he expected a Machian mass to emerge from his gravity theory [11], but after years of argument over things like the mass of a test particle in an otherwise empty universe he gave up on this view. However, in terms of coordinate velocity, it is clear that GR regulates light speed with regard to non-local observers. This is connected with time dilation on the one hand, which affects the coordinate velocity of all objects, and also with the spatial coefficient, which affects the coordinate velocity component aligned with the field gradient. So in effect the inertia-causing property is there, but discussing it in the context of invariant rest mass is prohibitively difficult.

From the inertia field perspective we will work around this using a concept of "coordinate inertia" in place of the locally invariant rest mass. Coordinate inertia is reference frame relative and used to formulate momentum relations, but does not affect the mass-energy sum of a collection of objects. In other words, the function of this kind of gravity-related inertia field (as opposed to the gravityindependent kind like Higgs) is to regulate the difficulty of moving (accelerating) an object with respect to another object or group of objects. It also cannot create mass out of nothing any more than can the gravity-independent inertia fields. But in regulating relative motion it takes on functions of gravity.

It is important to distinguish the inertia field approach from others which sound similar such as field gravity [12-14]. Field gravity has not been worked out beyond weak fields using time dilation and is 
presumed dependent on a background. It begins with the assumption gravity is a standard quantum force field. Versions so far seem to make predictions (e.g. on precession) that are not correct. By contrast, the framework we will devise uses fields to create either the actual space-time or the effects of space-time (a flexibility left to particular theories), and gravitational motion can be found in the usual way (by geodesics) if desired. The regulation of motion through momentum or inertia is the primary effect, not a force.

The framework conceptually does not assume any restrictions on topology even though for simplicity many of the conclusions are derived from one coordinate background. But it is not necessarily more than a coordinate system and not required to be a physical background (optionally it can be). Even within the background approach, a non-flat topology could be used as a background. Or, the field might generate space-time in any configuration to achieve the same level of flexibility as GR.

From a quantum field perspective, one presumes a new spin-2 boson will be required. But we do not go beyond a classical framework and do not specifically require this. The reason is that if there is a conflict between space-time gravity and vacuum-reaction inertia-gravity, then there may be the same conflict between spin-2 quantum field gravity and vacuum-reaction inertia-gravity. We leave these questions to the quantum field developers, with the exception that in a speculative discussion section we present a notion of degrees of freedom in regard to spin in the polarized vacuum that is different than in a real (non-virtual) spin-1 field. This is not part of our formal framework.

\section{Coordinate Inertia in a Homogeneous Reference}

When we transform a quantity or relation from local to observer coordinates in a way that preserves physical laws, we will say we have "coordinatized" it. To develop coordinate (or relative) inertia a homogeneous coordinate space will be used in which Noether's theorem may be applied [15], providing conservation of momentum and energy, though this means the effects of the gravitational field on momentum and energy have to be accounted for. There are two gravitational effects we are concerned with in this regard. First the action of gravitational acceleration on a mass transiting between different potentials in the field can be removed by using motion transverse to the field acceleration vector. This is not as limiting as it first seems, because we can transfer momentum using levers and rods without actually moving masses through potential energy differences in the field, as shown in Figure 1. Such devices are assumed to bend, contract or provide delay as required by relativity but to be lossless in momentum transfer.

The second gravitational effect on momentum of present concern is time dilation. It is easy to notice it because (barring singularities) broadcast signals can convey time intervals to other positions in the field for comparison. However, consider if we push or pull on a mass in a region of time dilation relative to the experimenter using a rod or tether. Its coordinate motion in the experimenter's frame is less than expected from Newton's law of motion. An object might even "slow down" in coordinate velocity as it enters the time dilated region, without any force applied, as does light, violating Newton's First Law of continuance of uniform motion in a coordinate space that conserves momentum and thus should uphold it.

In the context of gravity-related inertia fields and assigning properties of motion and resistance to motion to "inertia," it is helpful to use a nomenclature in which we say the "coordinate inertia" of objects changes, thus explaining the coordinate motion.

\section{$2.1 \quad$ Temporal Inertia Factor}

Again using Figure 1, two masses $m$ are identical and a vertically placed reaction bar and fulcrum transfers momentum from the top position 1 to the bottom 2. Radius to the top is sufficient that time dilation can be ignored there, for the sake of simplicity. The Schwarzschild metric for the setup is

$$
d s^{2}=-d \tau^{2} c^{2}=g_{t t} d t^{2}+g_{r r} d r^{2}+g_{\theta \theta} d \theta^{2}+g_{\phi \phi} d \phi^{2}
$$




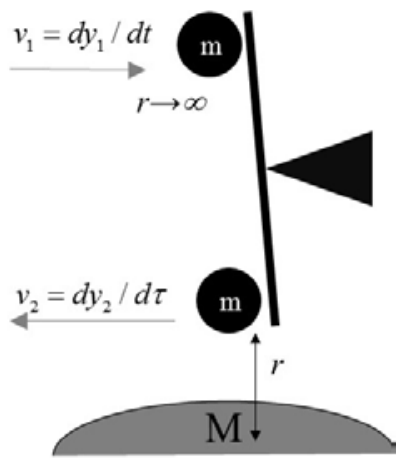

Figure 1. Momentum transfer using homogeneous coordinates (horizontal $d y$ ) in a gravitational field

We will dispense with the angle parts and instead use a small transverse tangent length $d y$ for the direction of motion of $m$ to meet our need for spatially homogeneous coordinates, at least in the direction where we wish to use conservation of momentum. We may assume $m$ is in a frictionless track to keep it from falling. The metric sign convention is -+++ . Extracting the time coefficient $g_{t t}=-c^{2}\left(1-2 G M / r c^{2}\right)$ we define the time dilation factor seen by a stationary distant observer at 1 :

$$
\begin{aligned}
& \varsigma=d t / d \tau=c /\left(-g_{t t}\right)^{1 / 2}=\left(1-2 G M / r c^{2}\right)^{-1 / 2} \\
& \Rightarrow d t=\varsigma d \tau
\end{aligned}
$$

\subsection{Conservation of Proper and Coordinate Momentum (CPM/CCM)}

The subscripts in Figure 1 tell us the local reference frame with respect to which a quantity is measured. To refer that quantity to another frame we'll use " $\rightarrow$ ", so for example $v_{2}=d y_{2} / d \tau \Rightarrow v_{2 \rightarrow 1}=d y_{2} / d t$. The momentum at 1 is then conserved in two ways. First it is conserved at 2 in local units so that $m v_{1}=m v_{2}$, which we call the conservation of proper momentum $(\mathrm{CPM})$ and from which we infer

$$
m v_{1}=m v_{2} \Leftrightarrow m d y_{1} / d t=m d y_{2} / d \tau=\varsigma m d y_{2} / d t=\varsigma m v_{2 \rightarrow 1}
$$

This is because the local coordinates are always homogeneous in that they are the same with respect to reference measurement standards (rods and clocks) transported to the local region. The coordinate view of $v_{2}$ from 1 is then reduced by the time dilation factor

$$
v_{2 \rightarrow 1}=v_{1} / \varsigma
$$

This is true even in the limit as $v_{1} \rightarrow c$ so that the coordinate speed of light as seen at 2 from 1 is reduced. The local speed measured with local (slow) clocks is of course still $c$.

Second, if we take the position of there being an inertia-causing field, then the mass at 2 can be said to have coordinate inertia $m_{2 \rightarrow 1}=\varsigma m_{2}$ from which we infer the conservation of coordinate momentum $(\mathrm{CCM})$ in homogeneous coordinates:

$$
m v_{1}=m_{2 \rightarrow 1} v_{2 \rightarrow 1}=(\varsigma m)\left(v_{1} / \varsigma\right)
$$

\subsection{Transitivity of Derivation and Assignment of Cause}

The above expressions were derived from a Schwarzschild metric as consequences of the GR view that gravity causes time dilation which causes the coordinate velocity effects. But if we are considering a gravity-related inertia field, then we should assume it is responsible for the coordinate inertia $\varsigma m$ and writing (5) as $m v_{1}=(\varsigma m) v_{2 \rightarrow 1}$ we would solve for coordinate velocity $v_{2 \rightarrow 1}=v_{2} / \varsigma$ and similarly work backward all the way to (3). Without a separate QFT "coordinate field equation" for the inertia-causing field, to replace Einstein's equation, then we still must use Schwarzschild for (2). But assuming we could produce an inertia-causing field of the correct intensity, it would cause all the same time related 
effects as gravity, which includes a good weak field approximation. For a long time this was the best anyone could do starting with inertia or a field (other than space-time). The purpose of pointing out this transitivity is to identify the method of maintaining consistency with GR. The framework is not supposed to introduce testable differences, but to establish criteria. A specific theory within the framework may, on the other hand, indicate a testable deviation at some point.

\subsection{Consistent Coordinate Field Strength (CFS) Postulate}

It is well known that the radial coordinate velocity of light is reduced by $\varsigma^{2}$. If from position 1 we apply a radial momentum impulse to a mass $m$ at position 2 , which we can do with the same apparatus followed by a 90 degree deflection, then we get less coordinate velocity than expected. This is a spatially anisotropic coordinate effect on motion and wavelength which does not affect time, since a clock will produce the same frequency regardless of orientation [16]. So the second factor of our framework, the spatial factor, affects coordinate lengths (thus coordinate velocity) but not time.

We can take the spatial factor from the Schwarzschild metric as we did for the temporal factor. Normally it is considered to be due to the field equation, and the coordinate laws of motion and equivalence alone are thought to be sufficient only to deduce the temporal factor, not the spatial factor. However, the spatial factor can be derived from a fairly intuitive postulate which makes it more clearly related to the inertia field, and might guide later attempts to develop a full inertia-gravity field theory.

Consider the setup of Figure 2 in which we can lower mass $m$ on a tether measuring the force at 1 , or drop it measuring the acceleration on the tether at 1 , either of which is a coordinate field strength. Assume the tether is massless and spools out exactly the same proper length $\delta l$ at the top as $m$ is lowered at the bottom. Further assume $\delta l$ is small enough we can treat the acceleration (if we were to let the tether spool freely) $g_{1}$ as constant. Then the coordinate force referenced to 1 with $m$ at any point $\mathrm{R}$ between $\mathrm{A}$ and $\mathrm{B}$, given that $g_{1}$ is constant, is

$$
\begin{aligned}
& F_{R \rightarrow 1}=m_{R \rightarrow 1} g_{R \rightarrow 1} \\
& F_{R \rightarrow 1}=\varsigma_{R} m g_{1}
\end{aligned}
$$

Even though acceleration is constant, there is a potential difference between A and B so time dilation is increasing between $\mathrm{A}$ and $\mathrm{B}$ and therefore $\varsigma_{R}$ increases toward B. It would appear that $m$ should be heavier even though the field strength (acceleration) hasn't changed. This is counter-intuitive. There is no obvious physical reason for it. It is like saying the mass $m$ if dragged behind a rocket would have more inertia on a longer tether (and in this sense the idea does follow from equivalence). Therefore, let us postulate consistent coordinate measures of field strength, whether acceleration or force, implying that over a constant field any remote coordinate measure of the field strength is also constant, or if they vary, then in the same manner.

Sticking with everything we've done so far, we have $\delta l$ left to work with. If we adopt a coordinate view of $\delta l$ varying inversely with time dilation, then we take the position that the coordinate $\delta l$ in 1's reference frame is $\delta l_{2 \rightarrow 1}=\delta l_{2} / \varsigma$, or more generally in terms of Schwarzschild coordinates:

$$
\begin{aligned}
& \varsigma=d r / d l_{\text {radial }}=g_{r r}{ }^{1 / 2}=\left(1-2 G M / r c^{2}\right)^{1 / 2} \\
& \Rightarrow d r=\varsigma d l_{\text {radial }}
\end{aligned}
$$

And so, there we have the spatial inertia factor, which in these coordinates is not independent of the temporal factor. They must compensate each other to preserve the consistency of coordinate field strength, which is in turn necessary for coordinate formulation of physical laws.

We will refer to the framework involving coordinate quantities and the consistent field strength (CFS) postulate as the CFS framework. In the results section we will apply it to exact GR solutions and give an example of a particularly simple inertia field direct solution. In the discussion section we will consider its degree of generality in connection with a conservative space-time principle. 


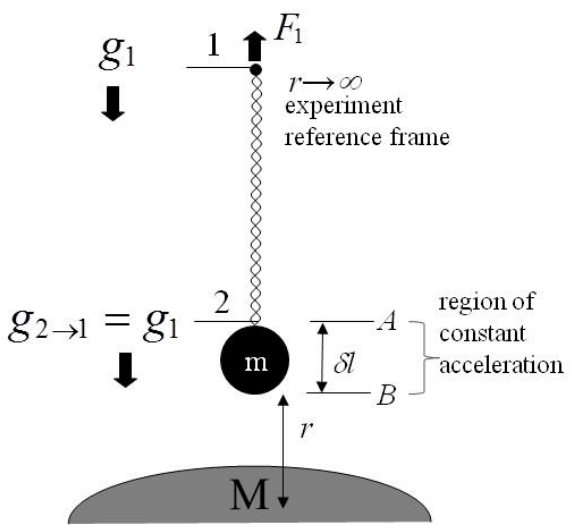

Figure 2. Measurement of coordinate field strength via tether

\section{$3 \quad$ Structure of the 2-Factor Field(s)}

For framework purposes we would prefer not to specify particular fields, such as the Higgs or the polarized (electromagnetic) vacuum. But we can look to them for suggestions about what kinds of properties an inertia-causing field is likely to have, and how to structure one in relation to a gravitational metric.

\subsection{The Planck Vacuum Intensity Can Produce Time Dilation}

The electromagnetic vacuum is a vector field with zero average value and thus on average no spatial density. It produces interactions due to the uncertainty in energy at short time intervals $d E d t>\hbar / 2$. Assuming a minimum $d E d t$ the inequality becomes an equation, with the energy available in a time interval $d \tau$ being $d E(d \tau) \approx \hbar / 2 d \tau$. The quantity $\hbar / 2$ is a locally constant scalar invariant which is fundamental to many quantum processes, so its constancy supports the equivalence principle. If measured in coordinate quantities (time, energy) it is not constant but is a ratio of energy to time which varies in a gravitational field. We will call this vacuum intensity. Applying the Schwarzschild metric for constant angular position, we find the coordinate relations:

$$
\begin{aligned}
& d E(d t) \approx \hbar / 2 d \tau \approx \hbar / 2\left(\left(-g_{t t}\right)^{1 / 2} / c\right) d t \\
& \Rightarrow d E(d t) d t \approx \hbar / 2\left(\left(-g_{t t}\right)^{1 / 2} / c\right) \\
& \Rightarrow d E(d t) d t / d E(d \tau) d \tau=1 /\left(\left(-g_{t t}\right)^{1 / 2} / c\right)=\varsigma
\end{aligned}
$$

If we apply this to the Planck relation $E=h f$ we infer $f=E / h$ in a local space-time region 2 . We can refer it to a distant coordinate observer 1 measuring time dilation $\varsigma_{2 \rightarrow 1}$ at 2 in this way:

$$
\begin{aligned}
& f_{2 \rightarrow 1}=f_{2} / \varsigma_{2 \rightarrow 1}=E_{2} / h \varsigma_{2 \rightarrow 1} \\
& \Leftrightarrow h_{2 \rightarrow 1}=h \varsigma_{2 \rightarrow 1}
\end{aligned}
$$

Thus the coordinate vacuum intensity $\varsigma \hbar / 2$ could be taken as a scalar field which produces time dilation, and whose gradient corresponds to the gravitational field strength. A path integral of the gradient gives time dilation or potential energy difference between two points, and also the spatial coordinate factor between the two points (in the direction of the gradient). The coordinatized Planck constant $h_{2 \rightarrow 1}$ is not used in our coordinate relationships, but is an alternate formulation introduced to support an analysis of full equivalence in the discussion section.

As the coordinate vacuum intensity is not energy, per se, it likely does not self-gravitate. The implied potential energy is negative and only manifests as associated with other mass-energy objects in the field. 


\subsection{The Spatial Field Requires an Anisotropic Factor}

We must also account for the spatial metric, which is anisotropic, or we do not have a complete account of gravity, only weak fields which are not conceptually definitive. As we have already concluded the Higgs can be independent of gravity, then it is assumed to be affected by whatever affects space and is not the cause. But it is as good as any other example to illustrate how space or the interactions that define space must be affected by whatever causes gravity, space-time or a spatial field with appropriate properties. We will also address questions about whether the Higgs field gravitates.

The Higgs field has a non-zero energy density, with no spin (or polarization), and acts as a condensate, keeping its state when one takes something out or puts something in. For that reason it cannot be "measured" directly. To do so would imply there is a definite quantity of it of which some is removed by the measurement, but we could continue removing forever, violating conservation of energy. Note that gravity can be measured, and so the Higgs field probably does not self-gravitate. For it to do so would imply it, like gravity, could be directly measured.

Generalizing so we are less tied to the Schwarzschild metric, let a volume in homogeneous coordinates be $d x d y d z$ and let $\rho \propto \varsigma^{-1}$ be the spatial inertia field density. This field must manifest anisotropy to support the spatial aspect of space-time. This it cannot do itself because a spin-0 field is not capable of anisotropy, so it must be linked with something that is capable. While the polarized vacuum at spin-1 is capable it is not sufficient. Spin-2 is expected for gravity to provide the degrees of freedom required by the tensor formulation.

Density of interactions, energy, mass, etc. whether measured locally or in observer coordinates is a scalar. However, we can define a component coordinate density of space to reflect the experience of a particle or wave group of certain local dimensions interacting along a coordinate length, capturing the spatial anisotropy which must be imposed to account for gravity. For framework purposes we do not specify whether it is (a) a density of something in space (e.g. interactions) which affects perception of space, (b) a density of space itself, or (c) merely a coordinate density due to mapping "expanded" space onto the fixed reference coordinates.

In Figure 3 the crosshairs represent spatial interaction density, and are not to be taken as discrete, but as an indicative scale. Density is progressively greater in the negative radial direction. Objects at A and $\mathrm{B}$ have local diameter $d s$. Observer coordinate units $d r$ and $d y$ are shown. The density per coordinate unit length if the object moves in a particular direction is the component coordinate density. For an object this size at A we can see that this is illustrated using $\rho_{r}=2 / d r=\rho_{y}=2 / d y$, but at B we have $\rho_{y}=2 / d y$ while $\rho_{r}=4 / d r$.

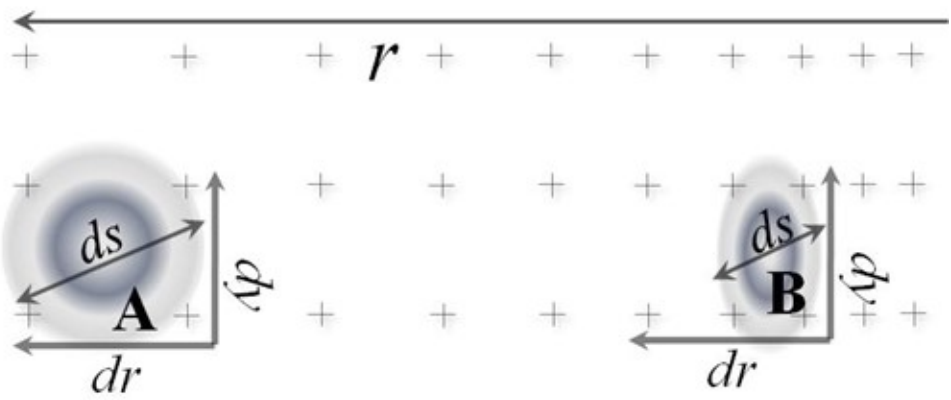

Figure 3. Illustration for component coordinate density

The spatial density field is thus not like a kinetic gas which equalizes its density in all directions. It is subject to some stress, like a stressed lattice of virtual (and relative) interactions, compressed in a certain direction by the linked field. That direction is the gradient of the temporal field, which is the gravitational acceleration vector.

We require two effects from this field. First, in any direction, the quantum field excitations that define objects have wavelengths proportional to the field density in that direction. Thus B appears 
radially compressed in coordinate space. Second, the coordinate momentum and inertia of objects is proportional to the cross section density of spatial field interaction points they encounter along directions of motion and acceleration. This is in addition to the time dilation effect. Thus A and B move transversely in a way which differs only by time dilation, since their transverse-motion spatial interaction cross sections are the same. But moved or accelerated radially, B responds with less coordinate velocity or acceleration because its radial-motion interaction cross section encounters anisotropic increased radial spatial interaction density.

\subsection{Combined Two-Factor Field Equation Using GR}

The principle work of making a classical field equation for the framework is to state a relationship between the gradient of the temporal field and the anisotropic spatial density field. To apply the CFS framework with the GR field relationships, the field quantities can be substituted in the diagonal of the metric in the GR field equation.

Let $\left(\rho_{x x}, \rho_{y y}, \rho_{z z}\right)$ represent ratios of the component coordinate densities between two different points $(x, y, z)_{1}$ and $(x, y, z)_{2}$ in the field, such that each component density ratio is the spatial coordinate ratio for that coordinate direction. Then we can write a GR metric in terms of our coordinate field strength ratios:

$$
\mathbf{g}_{\mu \nu}=\left[\begin{array}{cccc}
-c^{2} / \varsigma^{2} & 0 & 0 & 0 \\
0 & \rho_{x x}{ }^{2} & 0 & 0 \\
0 & 0 & \rho_{y y}{ }^{2} & 0 \\
0 & 0 & 0 & \rho_{z z}{ }^{2}
\end{array}\right]
$$

This metric when substituted into the Einstein field equation must give the same results as GR whenever the off-diagonal elements can be neglected.

\subsection{Example of an Inertia-Oriented Static Field Derivation}

The advantage of the CFS framework is that the spatial factor follows by conservation principles from time dilation. Any theory that recognizes the relation between time or frequency and potential energy, inevitable because gravity is universal (c.f. the electric field, with no net force when lowering a particleantiparticle pair into it, full energy is recovered from their annihilation), can produce weak field gravity through a Hamiltonian or Lagrangian. Now for the first time the spatial factor is also a consequence.

To illustrate how the CFS framework can be applied to new theories, with testable differences in extremely strong fields but otherwise conformal to GR, we formulate coordinate inertia as the direct primary effect of a field rather than an indirect consequence of space-time effects within the Schwarzschild solution.

Assume a field $\Phi$ which is under a stress $\Phi_{S}$ obeying Gauss' Law $\oint_{\partial V} \Phi_{S} d A=k_{\Phi}$ and Poisson's equation $\nabla \cdot \Phi_{S}=k_{\Phi} \mu$ where $\mu$ is source mass-energy density. For convenience assume point mass $\boldsymbol{M}$ and use Poisson only for the vacuum. However, in this case $\Phi_{S}$ is not force or acceleration but an as yet to be defined stress in a quantum field that will give rise to classical quantities through coordinates and measurement. This stress, by Gauss/Poisson, will have an inverse square form $\Phi_{S}=k / r^{2}$. A path

integral $\int_{\infty}^{r} \Phi_{S} d r=k_{\infty}+k / r$ represents the increase in coordinate inertia as we go from infinity where some test mass has background inertia $m=k_{\infty} / c^{2}$ to a radius $\boldsymbol{r}$ where it is increased by $\boldsymbol{k} / \boldsymbol{r}$. As we expect a greater coordinate inertia is required for time dilation, $\boldsymbol{k}$ is positive, not negative as usually the case with potential energy. Using the Newtonian limit and dividing by $c^{2}$ to get mass instead of energy gives $k=G M / c^{2}$. The coordinate inertia $\varsigma m$ in our previous terminology is the sum of the background and added components. Normalizing by $1 / m$ :

$$
\begin{aligned}
& \varsigma m / m=m / m+G M m / r c^{2} m \\
& \Rightarrow \varsigma=1+G M / r c^{2}
\end{aligned}
$$


From (7) we infer $\rho_{r r}=\varsigma^{-1}=\left(1+G M / r c^{2}\right)^{-1}$. These values are familiar as $2^{\text {nd }}$ order accurate approximations to the Schwarzschild metric, but in this example of an inertia-primary field development they are exact. The possibility of testing such a close theory has only recently been considered, but could be possible in the not too distant future [17]. A tensor field equation would be in terms of $\Phi$ and perceived or actual (depending on interpretation) local space-time would be emergent. Solving for the static inertia-oriented metric was intriguingly simple, not involving a system of simultaneous differential equations like those required for the Schwarzschild metric.

\section{Discussion}

Our formal results are concluded. This section is a somewhat speculative discussion of possible interpretations and mechanisms which are tentative and a guide for future inquiry.

\subsection{Vacuum Polarization vs. "Alignment"}

Since no known field has the required spin-2 property required by gravity, none of the fields currently being suggested as the source of inertia can be viewed as complete. It is assumed generally that if a spin2 field is found, it will be a graviton field associated with gravity. Then from the quantum field viewpoint this graviton would be responsible for the coordinate anisotropy of spatial fields (e.g. the Higgs scalar density field). From the metric gravity viewpoint it might be a sort of artifact of observing events of curved space-time in a homogeneous or flat coordinate system.

Here we note briefly a subject for possible further investigation. The polarized vacuum, as a consequence of being "virtual" in the sense of having virtual particle pairs coming and going over short intervals, has degrees of freedom that a non-virtual (real) field with persistent states (particles, momentum, charge, etc.) does not have. In Figure 4 a time series of virtual particle pairs are depicted at a point. These are not the only possible sequences. One might think of a Feynman like integral through possible paths in time.

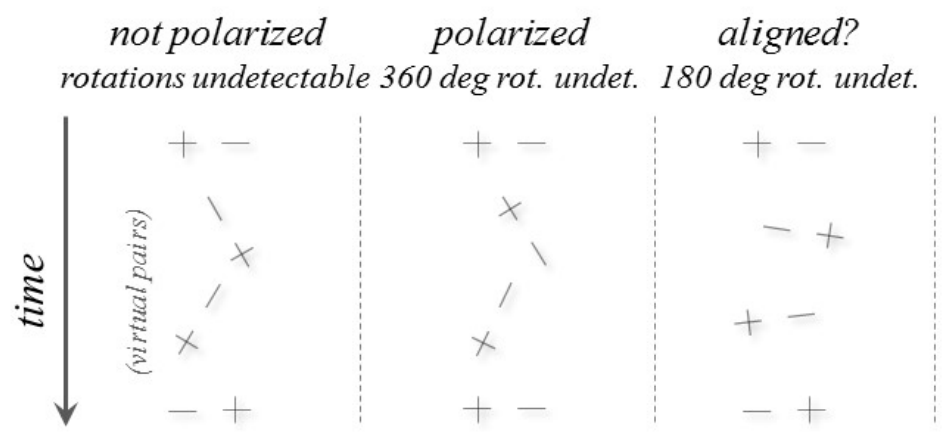

Figure 4. Vacuum (zero-point) polarization vs. alignment

Each particle pair which is a charge anti-charge pair has a polarization. In the absence of fields they must be dispersed in orientation so that the average polarization is zero, as on the left. In the middle is shown a conventional view of an electric field in which the vacuum pairs support and convey the field polarization via a statistical spatial orientation of charges in the direction of the field. On the right we have a clear spatial "alignment" of the charge pairs, favoring horizontal, but no E-field polarization. If this alignment is detectable or has physical effects, and we do not say that it does or does not, then it has an interesting property of invariance under 180 degree rotations and might be exploited to produce anisotropic effects.

\subsection{Full Equivalence and a Possible Planck Field}

Gravity linked to inertia-causing fields as in (10) clearly upholds weak equivalence. Given our 
assumption in discussing the temporal field that Planck's constant is locally constant, not really any different than assuming it is locally constant in curved space-time, many additional physical laws are included in the equivalence. Depending on whether one is using the CFS framework just to understand inertia-causing fields in a GR context, or to develop gravity from quantum fields on a background, more consideration than just an assumption might be due to deriving an appropriate coordinate value of Planck's constant. A reasonable possibility is to presume the coordinatized Planck constant $\varsigma h$ is the field and that time dilation is a consequence. The concept should probably be asked to handle other problems as well, such as producing and maintaining spatial anisotropy.

\subsection{Laws of Motion and Geometry}

We have covered sufficient ground to argue that inertia-derived space-time has the same laws of geodesic motion as GR. This provides no additional insight, which is something we might like to have, so a more detailed but informal discussion follows.

It has been shown elsewhere that coordinate light speed in homogeneous coordinates gives null geodesics to and beyond $2^{\text {nd }}$ order [16]. We can treat matter with rest mass as confined energy, e.g. photons in a box, which produces inertia [Ibid. 7-8] and whose trajectory is ultimately related to the photon trajectories already proved. Then we argue by equivalence that any other form of rest mass should have the same trajectory as an equal-energy box of photons, and that time-like geodesics are thus derived from null geodesics and equivalence. Then the laws of motion are satisfied constructively.

With QFT we now have an idea of all matter and energy as wave excitations in quantum fields. The frequency and speed of these waves is controlled by Planck's constant, thus time dilation is immediately and universally satisfied by QFT if the vacuum intensity hypothesis is accepted. Can we apply this to the spatial changes in lengths which also depend on Planck's constant? Only if we provide a spin-2 field and a mechanism to make the experience of the vacuum intensity anisotropic, so this is the major challenge for inertia-gravity field theory if one wishes to take it as primary and causal.

\subsection{Topology, Degrees of Freedom and the Conservative Space-Time Principle (CSP)}

A relation between the temporal and spatial factors was derived using homogeneous coordinates and a consistency of coordinate field strength measurement postulate (CFS). Note that homogeneous does not necessarily mean flat. The surface of a sphere is homogeneous, for example, even if finding a homogeneous coordinate system for it is non-trivial. Space-times which can be represented as homogeneous (flat or not, singular or not, expanding or not) and obey the CFS postulate can be described as conservative space-times.

In homogeneous coordinates, it is suggested by (7) the following product will be constant:

$$
k_{S T}=\rho_{x x} \rho_{y y} \rho_{z z} \varsigma
$$

In non-homogeneous coordinates it will have a value which is dependent only on the deviation from homogeneity, not anything physical. Not only is the space-time product conserved, it has other conservation implications. We choose to call this the conservative space-time principle (CSP).

Spherically symmetric space-times do not have the degrees of freedom to form topologies such as A or $\mathrm{B}$ in Figure 5, and are more often associated with topology $\mathrm{C}$ (which could be closed, though not shown as such). In $\mathrm{C}$ areas of space outside an arbitrary sphere cannot be reached from inside the sphere without contacting the sphere.

Though the CSP was derived under conditions like C, it does not necessarily limit topology and seems likely to apply more broadly based on the following informal argument. In GR the spatial part of the metric is path independent (though elapsed time is not), i.e. the clone of an object returning to its point of origin always agrees in dimensions with the original object. The CSP would require that also the time dilation factor return to the same value (not to be confused with elapsed time). The time dilation factor is associated with potential energy. Therefore from point $p$ in $\mathrm{A}$, the potential of $m$ is path independent, the same regardless of which mouth of the wormhole is used to access $m$. 


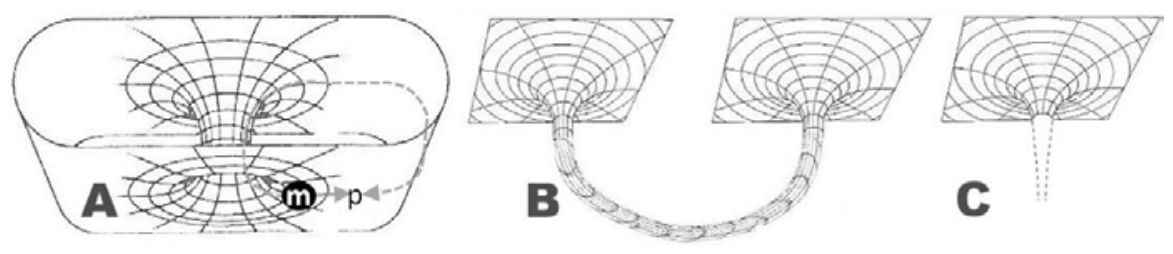

Figure 5. Conservative vs. merely symmetric space-times

This guarantees conservation of energy. And if we use indirect momentum transfer as in Figure 1 it also guarantees conservation of momentum. In either case we are assuming no evolution of the space while the experiment is underway. Obviously over time if the space expands or contracts, momentum and energy change. GR is not conservative in that way. But for a particular configuration of the space, the CSP would make it conservative.

\section{Conclusion}

The effort to find the properties of the Higgs field is of a magnitude with few parallels in history. If those properties meet fairly simple criteria, they will be independent of gravity as hoped. Further, those same criteria would assure the Higgs does not conflict with other proposed inertia-causing fields, such as a vacuum-reaction force, which are in fact gravity-related.

A gravity-related inertia field theory, one which affects momentum reactions, not just reallocation of mass-energy, is much more challenging. The CFS framework, derived largely from GR, reveals that a time dilation field must effectively be a Planck field, in coordinate space; otherwise the laws of physics are not preserved, and that the spatial field effects must result from conservation principles. The spatial field is left deliberately vague in the framework to avoid making it more than a framework, but it is clear that the anisotropy must be accounted for and must affect coordinate velocity and acceleration components along the field gradient, and coordinate measure of distances along the field gradient.

The CFS framework provides a high degree of compatibility with GR when using the field terms in the GR field equation, with some limitations since the framework does not specify dynamics (thus no off diagonal elements). It also supports generalization beyond GR, i.e. to allow a fields with different bases of effect as long as they follow the temporal-spatial relation and in consequence the principle of conservative space-time. An example static field derivation using inertia as the primary field with spacetime emergent showed that all alternatives need not necessarily be more complex than the GR field equation. Finally, a speculative discussion of degrees of freedom in the polarized virtual vacuum different from a non-virtual field suggests directions for further investigation.

Immediate further work will present the CFS framework in a vector formulation and derive the Schwarzschild metric exactly using only our coordinate relations (in lieu of tensors) and an appropriate Poisson/Gauss condition. The author does not have any plans to pursue quantum field theories, but encourages others to do so. It is hoped this framework will help clarify the relation between various investigations of inertia. In the $20^{\text {th }}$ century GR verification issues were largely astrophysical and cosmological. The framework could contribute to verification of strong field GR in the $21^{\text {st }}$ century by enabling comparison to a new class of conceptually sound counterpoint theories.

Disclaimer and Disclosure: The opinions in this paper do not necessarily represent those of the National Aeronautics and Space Administration. The author declares there are no conflicts of interest in regard to this work.

\section{References}

1. P.W. Higgs, "Broken symmetries, massless particles and gauge fields," Phys. Lett., 12 (132) (1964).

2. S. Chatrchyan, et. al., "Observation of a new boson at a mass of $125 \mathrm{GeV}$ with the CMS experiment at the LHC," Phys. Lett. B, 716 (1) 30-61 (2012). 
3. S. Weinberg, Gravitation and Cosmology: Principles and Applications of the General Theory of Relativity (Wiley and Sons, New York, 1972) vii-viii.

4. Rueda and B. Haisch, "Inertia as reaction of the vacuum to accelerated motion," Phys. Lett. A, 240 (3) 115 (1998).

5. H.E. Puthoff, "Polarizable-Vacuum (PV) Approach to General Relativity," Found. Phys. 32 (6) 927-943 (2002).

6. Rueda and B. Haisch, "Gravity and the quantum vacuum inertia hypothesis," Annalen der Physik, 14 (8) 479498 (2005).

7. M.B. van der Mark and G.W. "t Hooft, "Light is Heavy," Van A tot Q, NNV (Nov 2000) arXiv:1508.06478v1.

8. J.A. Macken, The Universe is Only Spacetime, (Macken, Santa Rosa, CA, 2015) revision 8, ch 1, p 1-11.

9. K. Jepsen, "Famous Higgs analogy, illustrated," Symmetry Magazine, Sept. 6 (2013) available at http://www.symmetrymagazine.org/article/september-2013/famous-higgs-analogy-illustrated.

10. R. Penrose, The Road to Reality, (Random House, London, 2004) pp. 628-632.

11. A. Einstein, "Is There a Gravitational Effect Which Is Analogous to Electrodynamic Induction?" Vierteljahrschrift für gerichtliche Medizin und öffentliches Sanitätswesen (ser. 3), 44, 37-40 (1912). Available in The Collected Papers of Albert Einstein, Vol. 4 (English), trans. Anna Beck, (Princeton University Press, Princeton, 1996).

12. W.E. Thirring, "An alternative approach to the theory of gravitation," Ann. Of Phys., 16, 96 (1961).

13. R. Feynman, Lectures on Gravitation 1962-63, (California Institute of Technology, Pasadena, CA, 1971).

14. Y. Baryshev, "Physics of Gravitational Interaction: Geometry of Space or Quantum Field in Space?" arXiv:grqc/0509105 (2005)

15. J. Hanc, S. Tuleja and M. Hancova, "Symmetries and conservation laws: Consequences of Noether's theorem," Am. J. Phys., 72 (4) 428-435 (2004).

16. R.L. Shuler, "Re-Visiting the Anisotropy of Inertia Experiments," AdAp, 1 (1) 47-53 (2016).

17. R.L. Shuler, "Numerical Geodesic Approximation for Theoretical and Experimental Light Bending Analysis," AdAp, 2 (1) 76-85 (2016). 